PDES, SUBMANIFOLDS AND

AFFINE DIFFERENTIAL GEOMETRY

BANACH CENTER PUBLICATIONS, VOLUME 57

INSTITUTE OF MATHEMATICS

POLISH ACADEMY OF SCIENCES

WARSZAWA 2002

\title{
ON NATURAL PARA-HERMITIAN STRUCTURES ON TWISTED PRODUCTS OF LIE GROUPS
}

\author{
ZBIGNIEW OLSZAK \\ Institute of Mathematics, Wroctaw University of Technology \\ Wybrzeże Wyspiańskiego 27, 50-370 Wrocław, Poland \\ E-mail: olszak@im.pwr.wroc.pl
}

\begin{abstract}
This survey article presents certain results concerning natural left invariant paraHermitian structures on twisted (especially, semidirect) products of Lie groups.

1. Preliminaries. Let $M$ be a connected $C^{\infty}$-differentiable manifold of even dimension $2 n$. All of the objects involved on $M$ are of class $C^{\infty}$ too. By $\mathfrak{X}(M)$ we denote the Lie algebra of vector fields on $M$.

By an almost paracomplex structure on $M$ we mean a $(1,1)$-tensor field $J$ on $M$ such that $J^{2}=\mathrm{Id}$ (the identity tensor field), and the eigendistributions $T^{ \pm} M$ associated to the eigenvalues \pm 1 of $J$, have the same dimension ([8], [6]). The manifold $M$ admits an almost paracomplex structure if and only if there exists a $G$-structure on $M$ with structural group $\mathrm{GL}(n, \mathbb{R}) \times \mathrm{GL}(n, \mathbb{R})$.

Let $J$ be an almost paracomplex structure on $M$ and $N_{J}$ be the Nijenhuis torsion tensor field of $J$,

$$
N_{J}(X, Y)=[J X, J Y]-J[X, J Y]-J[J X, Y]+J^{2}[X, Y], \quad X, Y \in \mathfrak{X}(M) .
$$

If $N_{J}$ vanishes identically on $M$, then $J$ is said to be paracomplex ([8]). Thus, the almost paracomplex structure $J$ is paracomplex if and only if the eigendistributions $T^{ \pm} M$ are completely integrable (in fact, this is a consequence of theorems concerning almost product structures by Walker [18] and Yano [19]), or equivalently, there exists a differentiable atlas of $M$ with coordinate maps satisfying the so-called para-Cauchy-Riemann equations $([4],[6])$.
\end{abstract}

If $g$ is a pseudo-Riemannian metric on $M$ for which the (almost) paracomplex structure $J$ is an antiisometry, that is,

$$
g(J X, J Y)=-g(X, Y), \quad X, Y \in \mathfrak{X}(M),
$$

2000 Mathematics Subject Classification: Primary 53C15; Secondary 53C50, 53B30.

Research partially supported by KBN grant 2P03A00617.

The paper is in final form and no version of it will be published elsewhere. 
then the pair $(J, g)$ is said to be an (almost) para-Hermitian structure on $M$ and the triple $(M, J, g)$ an (almost) para-Hermitian manifold ([8]). With respect to such a metric, the eigendistributions $T^{ \pm} M$ become isotropic. If $(J, g)$ is an almost para-Hermitian structure, then for any function $f$ on $M$, the pair $\left(J, \bar{g}=e^{2 f} g\right)$ is an almost para-Hermitian structure, too.

For an almost para-Hermitian manifold, the fundamental 2-form $\Omega$ (which is really an almost symplectic form) is defined by $\Omega(X, Y)=g(J X, Y)$ for $X, Y \in \mathfrak{X}(M)$.

An (almost) para-Hermitian manifold is said to be (almost) para-Kählerian if its fundamental form $\Omega$ is closed ([8]). Every 2-dimensional almost para-Hermitian manifold is para-Kählerian. An almost para-Hermitian manifold is para-Kählerian if and only if $\nabla J=0$, where $\nabla$ indicates the Levi-Civita connection of $g$.

We say that an almost para-Hermitian manifold $(M, J, g)$ is locally conformally paraKählerian if for any point $p \in M$ there exist a neighborhood $U$ of $p$ and a function $f: U \rightarrow \mathbb{R}$ such that $\left(J, e^{-2 f} g\right)$ is a para-Kählerian structure on $U$. An almost paraHermitian manifold $(M, J, g)$ is locally conformally para-Kählerian if and only if it is para-Hermitian and there is a 1 -form $\omega$ on $M$ satisfying the conditions ([5])

$$
d \Omega=2 \omega \wedge \Omega, \quad d \omega=0 .
$$

Note that when $\operatorname{dim} M=2 n \geq 6$, the condition $d \omega=0$ follows from $d \Omega=2 \omega \wedge \Omega$. If $\operatorname{dim} M=4$, the condition $d \Omega=2 \omega \wedge \Omega$ is automatically fulfilled with a certain 1-form $\omega$, however $d \omega \neq 0$ in general $([10])$.

The articles [2], [3] should be referred to for the generalities of the theory of almost para-Hermitian structures.

2. Twisted products of Lie groups. Let $G, H$ be Lie groups and assume that there exist Lie group homomorphisms

$$
\lambda: H \ni q \mapsto \lambda_{q} \in \operatorname{Aut}(G), \quad \mu: G \ni p \mapsto \mu_{p} \in \operatorname{Aut}(H) .
$$

In other words, $\lambda$ and $\mu$ are actions by automorphisms of $H$ on $G$ and $G$ on $H$, respectively. Assume additionally that

$$
\mu_{p}(q) q^{-1} \in \operatorname{Ker}(\lambda), \quad \lambda_{q}(p) p^{-1} \in \operatorname{Ker}(\mu)
$$

for any $p \in G, q \in H$. The twisted product $G \times_{\lambda, \mu} H$ is the Lie group defined in the following way: its underlying manifold is just the product manifold $G \times H$ and the multiplication is defined by

$$
\left(p_{1}, q_{1}\right)\left(p_{2}, q_{2}\right)=\left(p_{1} \lambda_{q_{1}}\left(p_{2}\right), q_{1} \mu_{p_{1}}\left(q_{2}\right)\right)
$$

for any $p_{i} \in G, q_{i} \in H, i=1,2$ (see [16]). The Lie group homomorphisms $\lambda, \mu$ induce, in an obvious way, the Lie algebra homomorphisms $A: \mathfrak{h} \rightarrow \operatorname{Der}(\mathfrak{g}), B: \mathfrak{g} \rightarrow \operatorname{Der}(\mathfrak{h})$ (op. cit.), which satisfy the following additional conditions

$$
A_{B_{X_{2}} Y} X_{1}-A_{B_{X_{1}} Y} X_{2}=0, \quad B_{A_{Y_{2}} X} Y_{1}-B_{A_{Y_{1}} X} Y_{2}=0
$$

for any $X, X_{1}, X_{2} \in \mathfrak{g}, Y, Y_{1}, Y_{2} \in \mathfrak{h}$.

The Lie algebra of $G \times_{\lambda, \mu} H$ is the twisted sum $\mathfrak{g} \oplus_{A, B} \mathfrak{h}$. Simplifying notations, we identify the linear space $\mathfrak{g} \oplus_{A, B} \mathfrak{h}$ with the direct sum of the linear spaces $\mathfrak{g}$ and $\mathfrak{h}$ and 
write an element $Z \in \mathfrak{g} \oplus_{A, B} \mathfrak{h}$ as the sum $Z=X+Y$ with $X \in \mathfrak{g}, Y \in \mathfrak{h}$. Then the Lie brackets in $\mathfrak{g} \oplus_{A, B} \mathfrak{h}$ are given by

$$
\left[X_{1}, X_{2}\right]=\left[X_{1}, X_{2}\right]_{\mathfrak{g}}, \quad[X, Y]=-[Y, X]=-A_{Y} X+B_{X} Y, \quad\left[Y_{1}, Y_{2}\right]=\left[Y_{1}, Y_{2}\right]_{\mathfrak{h}}
$$

for $X, X_{1}, X_{2} \in \mathfrak{g}$ and $Y, Y_{1}, Y_{2} \in \mathfrak{h}$. Since $A$ and $B$ are Lie algebra homomorphisms, we have additionally

$$
\begin{aligned}
A_{\left[Y_{1}, Y_{2}\right]_{\mathfrak{h}}} & =A_{Y_{1}} \circ A_{Y_{2}}-A_{Y_{2}} \circ A_{Y_{1}}=\left[A_{Y_{1}}, A_{Y_{2}}\right]_{\operatorname{Der}(\mathfrak{g})}, \\
B_{\left[X_{1}, X_{2}\right]_{\mathfrak{g}}} & =B_{X_{1}} \circ B_{X_{2}}-B_{X_{2}} \circ B_{X_{1}}=\left[B_{X_{1}}, B_{X_{2}}\right]_{\operatorname{Der}(\mathfrak{h})},
\end{aligned}
$$

and since $A_{Y} \in \operatorname{Der}(\mathfrak{g})$ and $B_{X} \in \operatorname{Der}(\mathfrak{h})$, it holds

$$
\begin{aligned}
A_{Y}\left[X_{1}, X_{2}\right]_{\mathfrak{g}} & =\left[A_{Y} X_{1}, X_{2}\right]_{\mathfrak{g}}+\left[X_{1}, A_{Y} X_{2}\right]_{\mathfrak{g}}, \\
B_{X}\left[Y_{1}, Y_{2}\right]_{\mathfrak{h}} & =\left[B_{X} Y_{1}, Y_{2}\right]_{\mathfrak{h}}+\left[Y_{1}, B_{X} Y_{2}\right]_{\mathfrak{h}}
\end{aligned}
$$

for any $X, X_{1}, X_{2} \in \mathfrak{g}, Y, Y_{1}, Y_{2} \in \mathfrak{h}$.

It is clear that in the case when

$$
\lambda_{q}=\operatorname{Id}_{G} \text { for any } q \in H, \quad \text { or } \mu_{p}=\operatorname{Id}_{H} \text { for any } p \in G,
$$

the twisted product becomes a semidirect product of Lie groups (for this notion see e.g. $[7])$; and in the case when

$$
\lambda_{q}=\operatorname{Id}_{G} \text { for any } q \in H, \quad \text { and } \mu_{p}=\operatorname{Id}_{H} \text { for any } p \in G,
$$

it is just the direct product of Lie groups. Note that for semidirect products we do not have to assume additionally (2) since it is always fulfilled in this case. To fix the convention in the rest of this article, a semidirect product will be denoted by $G \times{ }_{\lambda} H$ (assuming $\mu_{p}=\operatorname{Id}_{H}$ for every $p \in G$ ).

3. Natural para-Hermitian structures. Let $G \times_{\lambda, \mu} H$ be a twisted product Lie group with $\operatorname{dim} G=\operatorname{dim} H=n$.

Define a left invariant almost paracomplex structure $J$ on $G \times{ }_{\lambda, \mu} H$ by assuming

$$
\left.J\right|_{\mathfrak{g}}=-\left.\operatorname{Id}\right|_{\mathfrak{g}},\left.\quad J\right|_{\mathfrak{h}}=\left.\operatorname{Id}\right|_{\mathfrak{h}} .
$$

Since the eigendistributions $T^{-}=\mathfrak{g}, T^{+}=\mathfrak{h}$ are completely integrable, $J$ is paracomplex. Let $g$ be an arbitrary left invariant pseudo-Riemannian metric of signature $(n, n)$ on $G \times_{\lambda, \mu} H$ for which $\mathfrak{g}, \mathfrak{h}$ are both isotropic, i.e., $\left.g\right|_{\mathfrak{g}}=0$ and $\left.g\right|_{\mathfrak{h}}=0$. Then the pair $(J, g)$ is a left invariant para-Hermitian structure on $G \times{ }_{\lambda, \mu} H$.

Thus, we have:

TheOREm 1 ([14]). A twisted product Lie group $G \times_{\lambda, \mu} H$ with $\operatorname{dim} G=\operatorname{dim} H=n$ admits left invariant para-Hermitian structures.

Any para-Hermitian structure defined in the above way will be called a natural left invariant para-Hermitian structure on the twisted product $G \times_{\lambda, \mu} H$. In the sequel, we will consider only the para-Hermitian structures $(J, g)$ of this kind.

For simplicity and if it is not otherwise stated, $X, X_{1}, X_{2}, \ldots$ and $Y, Y_{1}, Y_{2}, \ldots$ will denote arbitrary elements of $\mathfrak{g}$ and $\mathfrak{h}$, respectively, while $Z, Z_{1}, Z_{2}, \ldots$ will be arbitrary elements of $\mathfrak{g} \oplus_{A, B} \mathfrak{h}$. 
The fundamental 2-form $\Omega$ corresponding to $(J, g)$ is given by

$$
\Omega\left(X_{1}, X_{2}\right)=0, \quad \Omega(X, Y)=-g(X, Y), \quad \Omega\left(Y_{1}, Y_{2}\right)=0 .
$$

The para-Hermitian metric $g$ and the Lie algebra homomorphisms $A, B$ enable us to define bilinear endomorphisms $A^{c}: \mathfrak{h} \times \mathfrak{h} \rightarrow \mathfrak{h}$ and $B^{c}: \mathfrak{g} \times \mathfrak{g} \rightarrow \mathfrak{g}$ by assuming that

$$
g\left(B_{X_{1}}^{c} X_{2}, Y\right)=g\left(B_{X_{1}} Y, X_{2}\right), \quad g\left(A_{Y_{1}}^{c} Y_{2}, X\right)=g\left(A_{Y_{1}} X, Y_{2}\right) .
$$

The following theorems characterize (up to locally conformal changes) para-Kählerian structures among natural para-Hermitian structures.

THEOREM 2 ([14]). Let $(J, g)$ be the natural left invariant para-Hermitian structure on a twisted product Lie group $G \times{ }_{\lambda, \mu} H$ with $\operatorname{dim} G=\operatorname{dim} H=n$.

$1)$ Let $n \geq 3$. The structure $(J, g)$ is locally conformally para-Kählerian if and only if there exists a left invariant 1 -form $\omega$ on $G \times_{\lambda, \mu} H$ such that

$$
\begin{aligned}
{\left[X_{1}, X_{2}\right] } & =-B_{X_{1}}^{c} X_{2}+B_{X_{2}}^{c} X_{1}-2 \omega\left(X_{1}\right) X_{2}+2 \omega\left(X_{2}\right) X_{1}, \\
{\left[Y_{1}, Y_{2}\right] } & =-A_{Y_{1}}^{c} Y_{2}+A_{Y_{2}}^{c} Y_{1}-2 \omega\left(Y_{1}\right) Y_{2}+2 \omega\left(Y_{2}\right) Y_{1} .
\end{aligned}
$$

The 1-form $\omega$ realizing (3), (4) satisfies also the following conditions

$$
\omega\left(B_{X_{1}}^{c} X_{2}\right)=\omega\left(B_{X_{2}}^{c} X_{1}\right), \quad \omega\left(B_{X} Y\right)=\omega\left(A_{Y} X\right), \quad \omega\left(A_{Y_{1}}^{c} Y_{2}\right)=\omega\left(A_{Y_{2}}^{c} Y_{1}\right) .
$$

2) Let $n=2$. There exists a left invariant 1 -form $\omega$ on $G \times_{\lambda, \mu} H$ such that (3) and (4) are fulfilled. The structure $(J, g)$ is locally conformally para-Kählerian if and only if the 1-form $\omega$ satisfies the conditions (5).

It should be added that the form $\omega$ occurring in the above theorem is just the form realizing (1). The theorem below is a direct consequence of the previous one.

THEOREM 3 ([14]). Let $(J, g)$ be the natural left invariant para-Hermitian structure on a twisted product Lie group $G \times_{\lambda, \mu} H$. The structure $(J, g)$ is para-Kählerian if and only if

$$
\left[X_{1}, X_{2}\right]=-B_{X_{1}}^{c} X_{2}+B_{X_{2}}^{c} X_{1}, \quad\left[Y_{1}, Y_{2}\right]=-A_{Y_{1}}^{c} Y_{2}+A_{Y_{2}}^{c} Y_{1} .
$$

As a consequence of the above two theorems, it is possible to formulate necessary and sufficient conditions for a natural para-Hermitian structure on a semidirect product of Lie groups to be (locally conformal) para-Kählerian (see also [11]).

\section{Para-Hermitian structures on twisted products of generalized Heisen-} berg groups. Let $\mathcal{H}^{2 k+1}, k \geq 1$, be the generalized Heisenberg group consisting of the matrices of the form

$$
\left[\begin{array}{cccccc}
1 & x^{1} & x^{2} & \cdots & x^{k} & x^{2 k+1} \\
0 & 1 & 0 & \cdots & 0 & x^{k+1} \\
0 & 0 & 1 & \cdots & 0 & x^{k+2} \\
\vdots & \vdots & \vdots & \ddots & \vdots & \vdots \\
0 & 0 & 0 & \cdots & 1 & x^{2 k} \\
0 & 0 & 0 & \cdots & 0 & 1
\end{array}\right]
$$

where $x^{1}, \ldots, x^{k}, x^{k+1}, \ldots, x^{2 k}, x^{2 k+1}$ are arbitrary real numbers. $\mathcal{H}^{2 k+1}$ is a connected simply-connected 2 -step nilpotent Lie group of dimension $2 k+1$ (see e.g. [1]). 
Consider the twisted products $G \times_{\lambda, \mu} H$, where $G=H=\mathcal{H}^{2 k+1}$ and $\lambda, \mu$ are the Lie group actions by the interior automorphisms, that is,

$$
\lambda_{y}(x)=y \cdot x \cdot y^{-1}, \quad \mu_{x}(y)=x \cdot y \cdot x^{-1}, \quad x \in G, y \in H .
$$

These twisted products are also 2-step nilpotent Lie groups [16].

For such twisted products, we have the following theorem.

THEOREM 4 ([14]). Let $(J, g)$ be the natural left invariant para-Hermitian structure on the twisted product $\mathcal{H}^{2 k+1} \times_{\lambda, \mu} \mathcal{H}^{2 k+1}$, where $\lambda, \mu$ are the actions by the interior automorphisms. If $k=1$ and $(J, g)$ is locally conformally para-Kählerian, then it is necessarily para-Kählerian. If $k \geq 2$, then $(J, g)$ is never locally conformally para-Kählerian.

In [14] it was also shown that it is possible to construct para-Kählerian structures on $\mathcal{H}^{3} \times{ }_{\lambda, \mu} \mathcal{H}^{3}$.

5. Other examples $([14])$. Let $\mathcal{K}^{n}$ the connected simply-connected non-Abelian solvable Lie group consisting of the matrices of the form

$$
\left[\begin{array}{cccc}
e^{-x^{1}} & x^{2} & \ldots & x^{n} \\
0 & 1 & \ldots & 0 \\
\vdots & \vdots & \ddots & \vdots \\
0 & 0 & \ldots & 1
\end{array}\right]
$$

where $x^{1}, x^{2}, \ldots, x^{n}$ are arbitrary real numbers. The underlying manifold of $\mathcal{K}^{n}$ is just the Cartesian space $\mathbb{R}^{n}$.

It can be shown that there exists a left invariant 1-form $\phi$ on $\mathcal{K}^{n}$ such that

$$
[X, Y]=-\phi(X) Y+\phi(Y) X, \quad X, Y \in \mathfrak{k}^{n},
$$

where $\mathfrak{k}^{n}$ denotes the Lie algebra of $\mathcal{K}^{n}$.

Let $G=H=\mathcal{K}^{n}$ and $\lambda: H \rightarrow \operatorname{Aut}(G), \mu: G \rightarrow \operatorname{Aut}(H)$ be the Lie group homomorphisms defined by

$$
\lambda_{y}(x)=\left(x^{1}, x^{2} e^{a y^{1}}, \ldots, x^{n} e^{a y^{1}}\right), \quad \mu_{x}(y)=\left(y^{1}, y^{2} e^{b x^{1}}, \ldots, y^{n} e^{b x^{1}}\right)
$$

for any $x \in G, y \in H$, where $a, b$ are certain fixed real constants. The conditions

$$
\lambda_{y}(x) \cdot x^{-1} \in \operatorname{Ker}(\mu), \quad \mu_{x}(y) \cdot y^{-1} \in \operatorname{Ker}(\lambda)
$$

are clearly fulfilled for any $x \in G, y \in H$. Thus, we have defined the twisted product $G \times{ }_{\lambda, \mu} H$.

Let $\phi, \psi$ be the left invariant 1-forms on $G, H$, for which

$$
\begin{aligned}
{\left[X_{1}, X_{2}\right] } & =-\phi\left(X_{1}\right) X_{2}+\phi\left(X_{2}\right) X_{1}, \quad X_{1}, X_{2} \in \mathfrak{g}, \\
{\left[Y_{1}, Y_{2}\right] } & =-\psi\left(Y_{1}\right) Y_{2}+\psi\left(Y_{2}\right) Y_{1}, \quad Y_{1}, Y_{2} \in \mathfrak{h} .
\end{aligned}
$$

The Lie algebra homomorphisms $A: \mathfrak{h} \rightarrow \operatorname{Der}(\mathfrak{g}), B: \mathfrak{g} \rightarrow \operatorname{Der}(\mathfrak{h})$ are given by

$$
A_{Y} X=a \psi(Y)(X-\phi(X) E), \quad B_{X} Y=b \phi(X)(Y-\psi(Y) F)
$$

for any $X \in \mathfrak{g}, Y \in \mathfrak{h}$, where $E \in \mathfrak{g}$ and $F \in \mathfrak{h}$ are such that $\phi(E)=1$ and $\psi(F)=1$.

Let $(J, g)$ be an arbitrary natural left invariant para-Hermitian structure on $G \times{ }_{\lambda, \mu} H$, for which $\phi(\cdot)=g(\cdot, F)$ and $\psi(\cdot)=g(\cdot, E)$ additionally. 
It is verified in [14] that the structure $(J, g)$ is locally conformally para-Kählerian with the form $\omega$ (cf. Theorem 2) given by

$$
\left.\omega\right|_{\mathfrak{g}}=\frac{1}{2}(1-b) \phi,\left.\quad \omega\right|_{\mathfrak{h}}=\frac{1}{2}(1-a) \psi .
$$

We see that the structure is para-Kählerian if and only if $a=b=1$.

6. Curvature conditions. With respect to the conformal flatness of natural paraHermitian structures, we have obtained the following results, which concern semidirect products only.

TheOREm 5 ([12], [13]). Let $(J, g)$ be a natural left invariant para-Hermitian structure on a semidirect product of Lie groups $G \times{ }_{\lambda} H$ with $\operatorname{dim} G=\operatorname{dim} H=2$. Then the metric $g$ is conformally flat if and only if the structure $(J, g)$ is locally conformally paraKählerian.

THEOREM 6 ([12]). If a natural left invariant para-Hermitian structure $(J, g)$ on a semidirect product of Lie groups $G \times{ }_{\lambda} H$ with $\operatorname{dim} G=\operatorname{dim} H \geq 3$ is locally conformally para-Kählerian, then the metric $g$ is conformally flat.

The assertions of Theorems 5 and 6 are not valid in the class of all para-Hermitian manifolds. Indeed, the existence of para-Kählerian manifolds which are not conformally flat is rather obvious. For concrete examples, one can consult for instance [15], where nonconformally flat para-Kählerian manifolds of recurrent conformal curvature are studied.

The converse to Theorem 6 does not hold in general. Namely, in [13] we have established the existence of a natural left invariant locally conformally para-Kählerian structure on a semidirect product of $\mathbb{S} U(2)$ and $\mathbb{R}^{3}$, which is not conformally flat.

In [12], we have also studied various curvature conditions of natural left invariant locally conformally para-Kählerian structures on semidirect products of Lie groups. We determined necessary and sufficient conditions for such structures to be flat as well as locally symmetric or semisymmetric in the sense of [17]. Among other results, we proved that if a natural left invariant para-Hermitian structure is para-Kählerian, then this is flat; and that the converse is not true in general. Moreover in that paper, various examples of natural left invariant locally conformally para-Kählerian structures realizing these curvature conditions were constructed on semidirect products $\mathbb{R}^{n} \times{ }_{\lambda} \mathbb{R}^{n}, \mathbb{R}^{n} \times{ }_{\lambda} \mathcal{K}^{n}$, $\mathcal{K}^{n} \times{ }_{\lambda} \mathbb{R}^{n}$ and $\mathcal{K}^{n} \times{ }_{\lambda} \mathcal{K}^{n}$, where $\mathcal{K}^{n}$ is the Lie group defined in Section 5 .

It is still not known whether the assertions of Theorems 5, 6 and the other theorems from paper [12] remain true if we replace the semidirect products by twisted products. Investigations about this problem will be continued by the author in the future.

Finally let us recall that conformally flat para-Kählerian manifolds (the general case) have already been classified from the local point of view in [9].

\section{References}

[1] J. Berndt, F. Tricerri and L. Vanhecke, Generalized Heisenberg Groups and Damek-Ricci Harmonic Spaces, Springer-Verlag, Berlin-Heidelberg, 1995. 
[2] V. Cruceanu, P. Fortuny and P. M. Gadea, A survey on paracomplex geometry, Rocky Mountain J. Math. 26 (1996), 83-115.

[3] V. Cruceanu, P. M. Gadea and J. Muñoz Masqué, Para-Hermitian and para-Kähler manifolds, Quaderni Inst. Mat., Univ. Messina 1 (1995), 1-72.

[4] A. Crumeyrolle, Variétés différentiables à structure complexe hyperbolique. Application à la théorie unitaire relativiste des champs, Riv. Mat. Univ. Parma (ser. 2 ) 8 (1967), 27-53.

[5] P. M. Gadea and J. Muñoz Masqué, Classification of almost para-Hermitian manifolds, Rend. Mat. Roma (Ser. 7) 11 (1991), 377-396.

[6] S. Kaneyuki and M. Kozai, Paracomplex structures and affine symmetric spaces, Tokyo J. Math. 8 (1985), 81-98.

[7] A. W. Knapp, Lie Groups Beyond an Introduction, Progr. Math. 140, Birkhäuser, Boston, 1996.

[8] P. Libermann, Sur le problème d'équivalence de certaines structures infinitésimales, Ann. Mat. Pura Appl. 36 (1954), 27-120.

[9] Z. Olszak, On conformally flat parakählerian manifolds, Math. Balkanica (N.S.) 5 (1991), 302-307.

[10] Z. Olszak, Four-dimensional para-Hermitian manifolds, Tensor (N.S.) 56 (1995), 215-226.

[11] Z. Olszak, Left invariant para-Hermitian structures on semidirect products of Lie groups, Tensor (N.S.), in print.

[12] Z. Olszak, On the curvature of left invariant locally conformally para-Kählerian metrics, in: Geometry and Topology of Submanifolds, Vol. IX, World Sci., Singapore, 1999, 198207.

[13] Z. Olszak, On 4-dimensional conformally flat left invariant para-Hermitian structures, Univ. Iagell. Acta Math. 38 (2000), 29-40.

[14] Z. Olszak, On para-Hermitian structures on twisted products of Lie gruops, Bull. Math. Soc. Sci. Math. Roumanie 43 (93) (2000), 313-323.

[15] Z. Olszak, On para-Kählerian manifolds of recurrent conformal curvature, Rend. Sem. Mat. Messina, in print.

[16] M. A. Rudkovskiı̌, The twisted product of Lie groups, Sibirsk. Mat. Zh. 38 (1997), 11201129 (in Russian).

[17] Z. I. Szabó, Structure theorems on Riemannian manifolds satisfying $R(X, Y) R=0$, I, Local version, J. Diff. Geom. 17 (1982), 531-582; II, Global versions, Geom. Dedicata 19 (1985), 65-108.

[18] A. G. Walker, Connections for parallel distribution in the large, Quart. J. Math. (Oxford) 6 (1955), 301-308.

[19] K. Yano, Affine connections in almost product spaces, Kodai Math. Sem. Rep. 11 (1959), $1-24$. 